\title{
Improving Efficiency and Effectiveness in Managed Care: Ongoing Efforts at the University of New Mexico College of Pharmacy
}

The state of New Mexico presents a mature and extremely competitive managed care market. The managed care share of the health care market in New Mexico has grown over the years and recently has included some of the Medicaid population. In 1997, three major managed care companies bid for a contract to manage and deliver health care to New Mexico Medicaid enrollees

In the Medicaid managed care scenario, appropriate drug utilization is very important to ensure the cost-effective use of medications. To this end, the New Mexico Drug Education and Utilization Review Board in Santa Fe has contracted with the University of New Mexico College of Pharmacy to run a Drug Utilization Review (DUR) Program.

This program is active in mailing intervention packets to both physicians and pharmacists in an attempt to correct prescribing behaviors. For example, the program regularly sends intervention letters to physicians and pharmacists for patients for whom inappropriate asthma therapy is detected. The drug utilization database that has been made available to the program is also being used for pharmacoeconomic evaluations.

One study is investigating the economic impact of the introduction of Salmeterol into the Medicaid formulary for the treatment of moderate to severe asthmatics. The Medicaid DUR Program is directed by H.William Kelly,

Pharm.D.; Melanie Dodd, Pharm.D., serves as the clinical pharmacist; and Gireesh V. Gupchup, Ph.D., serves as a consultant to the program.

Gupchup and Matthew Borrego, Ph.D., at the University are working with Rich Reynolds, R.Ph., at Lovelace Health Systems, a large managed care organization in Albuquerque, to compare the differences between two alternative aminoglycoside dosing protocols in clinical effectiveness, nephrotoxicity, and cost effectiveness. Though the literature suggests that once-daily dosing is considerably less costly than multiple-daily dosing, preliminary results from the Lovelace Medical Center seem to indicate that a multiple daily dosing protocol with a Clinical Pharmacokinetic Service (CPS) is slightly more cost effective.

Morgan Witter, Pharm.D., and Gupchup have completed another project with implications for managed care organizations that compared the use of enoxaparin in the skilled nursing ward of a long-term care facility (LTCF) with the use of unfractionated heparin in a hospital for the treatment of proximal deep vein thrombosis in geriatic patients. From the managed care perspective, using enoxaparin in the LTCF is a more costeffective option in terms of total expense per successful therapy, constitutes less disruptive treatment, and obviates the need to remove the patient from familiar surroundings. Sensitivity analyses showed that the decision analysis model was robust to certain key alterations.

Other DUR projects also might have an impact on the efficiency and effectiveness of managed care. Puneet Singhal, B.S., a graduate student in pharmacy administration, under the supervision of Dennis Raisch, Ph.D., of the Veterans Administration Cooperative Studies Program, is investigating health outcomes associated with substance abuse treatment and detoxification programs. Another study by Gupchup, Singhal, Ernest J. Dole, Pharm.D., and Buford T. Lively, Ed.D., on burnout among HMO pharmacists was reported in the September/ October 1998 issue of JMCP. Finally, Gupchup, Joseph Hubbard, Pharm.D., and other. researchers at the College of Pharmacy, in collaboration with pharmacists in the Albuquerque Service Unit of the Indian Health Service, are developing an asthma-specific quality-of-life questionnaire for Native American adults; there currently is no culturally relevant instrument for this purpose. The questionnaire is for use as an outcome measure in an asthma pharmaceutical care plan in the Albuquerque Service Unit of the Indian Health Service.

As managed care becomes more widespread in New Mexico and nationwide, the College of Pharmacy is initiating an increasing number of projects related to managed care issues. The Division of Pharmacy Administration at the college is sharply focused on. improving health outcomes in the managed care population and is training many students being trained at the M.S and Ph.D. levels to accomplish this.

Gireesh V. Gupchup, Ph.D. Assistant Professor of Pharmacy and Concentration Chair

Pharmacy Administration Graduate Program University of New Mexico

Health Sciences Center College of Pharmacy Albuquerque, NM 